\title{
Moliniasis vulvo-vaginal
}

\author{
Por el doctor Hernando Amaya León
}

Profesor Agregado de Ginecología. Facultad de Medicina de la Universidad Nacional.

Del Departamento de Obstetricia y Ginecología del "Consultorio de Especialistas"

\section{I.-ANOTACIONES SOBRE CRITERIOS DIAGNOSTICOS Y DE CURACION}

Por tener la intención de exponer en los próximos capítulos algunos puntos referentes a incidencia y tratamiento de la moniliasis vulvo-vaginal en nuestro medio, me ha parecido de capital importancia discutir antes el criterio personal que he adquirido en relación a diagnóstico y al término "curación".

Revisando la totalidad de la literatura a mi alcance a este respecto, no pude encontrar similitud de pareceres; hay opiniones semejantes en muchos aspectos, pero también las hay ampliamente discordantes. De su estudio y mi experiencia clínica he creído deducir algo que pueda ayudar para aclarar estos conceptos.

Tomando a Dubos (1) en su excelente tratado, resumimos que “. . Candida Albicans es un hongo levaduriforme oval, que produce blastoporos y pseudomicelios en los tejidos y exudados, y en cultivos a temperatura ambiente y a 37 grados. $\mathrm{Su}$ significado etiológico exacto en determinada enfermedad es difícil de establecer, pues se encuentra con frecuencia en bocas y tractos intestinales normales, o como contaminación secundaria en otras enfermedades conocidas". Luego, “...las especies Candida son habitantes normales en boca, intestino y vagina, y pueden cultivarse de estas localizaciones en 35 a $40 \%$ de los individuos normales...", y otros autores, entre ellos Swartz (2) destacan que "...los organismos Monilia, sin embargo, pueden habitar en la región vaginal como saprofitos...".

Volviendo a Dubos “... solamente Candida Albicans es patogénica para animales de laboratorio; los conejos inyectados intravenosamente con 1 c. c. de una suspensión salina, mueren 4 o 5 días después con abscesos típicos del riñón". Tratando de inmu- 
nología afirma que el 40 a $50 \%$ de los adultos muestran positividad al test cutáneo de la vacuna de Candida Albicans o a la oidiomycina, lo cual le concede un significado diagnóstico muy dudoso. Por otra parte, el $45 \%$ de los sueros humanos normales aglutinan con Candida Albicans, y esto indica claramente que los tests de aglutinación tienen un escasísimo valor.

Como procedimientos útiles en el diagnóstico quedan entonces los exámenes de frotis en fresco, las coloraciones del tipo Gram, y los cultivos en medio de Saboureaud adicionados de glucosa y agar, los cuales prenden en 4 o 5 días a la temperatura ambiente y en 24-48 horas a la temperatura de 37 grados. Actualmente se aconsejan medios con antibióticos.

He deseado insistir en estos puntos porque he oído varias veces el concepto en algunos médicos, y sobre todo en laboratoristas clínicos, de que no es posible admitir el diagnóstico de moniliasis vaginal si no está precedido por un cultivo, y esto debe ser discutido.

Algunos textos afirman que el diagnóstico de Candida por examen de frotis en fresco es muy difícil y es realidad, sobre todo para "... aquellos faltos de experiencia en el examen de frotis vaginales en fresco..." (3). En la corporación en donde trabajo (Consultorio de Especialistas), contamos con la colaboración de uno de los más expertos laboratoristas, el doctor F. Cortés, quien a la vez es un médico dotado de excelente criterio clínico; y puedo asegurar que, salvo en muy escasas ocasiones, hemos necesitado recurrir a otros procedimientos distintos de los exámenes en fresco para hacer la demostración del organismo Candida en secreciones vulvo-vaginales.

Por otra parte, todos los autores extranjeros estudiados están de acuerdo en la predominancia casi absoluta de la especie Albicans; así Campbell y Parrot (4), cultivando secreciones vaginales en medio de Saboureaud adicionado de 15 unidades de penicilina, encuentran 133 casos de Monilia Albicans en 166 cultivos positivos, o sea el $80 \%$; Bernstein y Rakoff (3) llegan al $89 \%$, y así sucesivamente. En nuestro laboratorio, para evitar trabajo no práctico y eximir al paciente de costos adicionales que consideramos inútiles, en presencia de monilias en el frotis húmedo se contesta como positivo al género Monilia, dando como casi seguro cie que se trate de la especie Albicans.

Ahora bien: analizando los puntos expuestos, es posible ver que:

1. Si una paciente consulta por flujo vaginal con caracteres 
clínicos predominantes de irritación y prurito casi siempre intenso (este síntoma es aún más molesto que en la tricomoniasis), y se encuentran organismos del género Candida en los frotis en fresco con exclusión de otros organismos tanto en éstos como en tinciones, se hace imperativo el diagnóstico de moniliasis. Tan sólo en los casos dudosos o negativos con clínica sospechosa debe ordenarse cultivo.

2. Si hay evidencia de diabetes, embarazo, y sobre todo antecedentes de ingestión de antibióticos o aplicación vaginal local de ellos, el diagnóstico no debe dudarse.

3. Si con las anteriores circunstancias se encuentra la Monilia asociada a Tricomonas u otros gérmenes, se deja preferencia al tratamiento de la micosis; si no es así, parece mejor iniciar el de la entidad asociada, pues con bastante frecuencia las drogas usadas son favorables a la exaltación de las monilias; pero en estas condiciones debe rechazarse el uso de antibióticos.

4. Si las condiciones físicas del exudado vaginal, y sobre todo los caracteres clínicos de la paciente no corresponden a las usuales de la moniliasis, en presencia de un frotis o cultivo positivo es preferible insistir en la búsqueda de otras causas etiológicas probables, por saberse que tal organismo es muchas veces un simple saprofito vaginal.

5. No importa, o importa poco en la práctica, determinar exactamente la especie de la monilia; baste saber que la encontrada es patógena, pues está produciendo sintomatología clínica suficiente, y que la que sea es sensible a los tratamientos usuales en la gran mayoría de los casos.

Lo antedicho es nuestro criterio en materia de diagnóstico. Con estos datos es posible evitar confusiones y aun situaciones contradictorias.

Ahora bien: en lo referente a curación, valen también los mismos puntos expuestos. Lo ideal, claro está, es determinar la ausencia de los organismos en los frotis vaginales. En los datos estadísticos que figuran en el capítulo sobre tratamientos, se aprecia que aquello es posible en el 70 al $85 \%$ de los casos. Pero desde Hesseltine y Beckette (5), Hart y Brown (6), y recientemente Henriksen y col. (7), debe admitirse que no es práctico establecer la curación, por intermedio de los cultivos negativos, sino considerar a la paciente "clínicamente curada" si presenta una cavidad vaginal nor- 
mal en varios exámenes y está asintomática por un período no menor de un mes. $Y$ estas normas son consecuencia lógica del recuerdo de que las monilias pueden ser habitantes saprofitos en condiciones normales.

Entonces podemos decir que una paciente está curada si:

1. Es asintomática y los exámenes de laboratorio son negativos; caso ideal.

2. Cuando hay exámenes vaginales repetidos, la cavidad se encuentra normal, y no hay sintomatología durante un mes, aun cuando el laboratorio sea positivo.

3. Si a pesar de los tratamientos la enferma presenta sintomatología atenuada y no hay evidencia de organismos en los exámenes en fresco y tintoriales, caso en el cual deberán hacerse cultivos. Si éstos son negativos, la enferma podrá ser considerada "curada", e insistir en la búsqueda de otras causas asociadas.

Para terminar, es bueno insistir en la posibilidad de encontrar otros focos micóticos en el resto del organismo, caso en el cual es imperativo tratarlos enérgicamente para evitar posibilidades infectantes. Así mismo, es conveniente examinar al marido, causa ccasional de reinfecciones.

No está por demás advertir a la paciente que ha sufrido de moniliasis vulvo-vaginal el peligro de usar antibióticos sin justificación, como es usual hoy en día, por el riesgo de micosis y sus consecuencias. Es obvio que en diabéticas el tratamiento de esta condición es básico, y en embarazadas, insinuar cuidados muy estrictos durante el resto de la gestación, lo mismo que en futuras situaciones similares.

\section{Ir.- CONTRIBUCION AL ESTUDIO DE SU INCIDENCIA EN NUESTRO MEDIO}

Desde Wilkinson (8) en 1840 , quien parece que fue el primero en describir este tipo de enfermedad, pasaron más de 100 años sin que se concediera mayor importancia a la localización de monilias en el tracto genital femenino. Aun más, fueron muchos los Que no creían en su existencia como enfermedad humana. Parece que su aceptación como tal no data sino de 1937 cuando Bland, Rakoff y Pinkus (9), por una parte, y Hesseltine (10) por otra, lograron comprobar la moniliasis vaginal en forma experimental. Sin embargo, al revisar textos modernos $(11,12)$, vemos que se le 
concede mínima importancia: Wharton en la edición de su libro de 1944 dice que "...es una variedad rara de infección vaginal y cervical". No es aventurado afirmar que esta entidad ha invadido el campo de la ginecologia en los últimos 8 a 10 años, y así lo dejan entender los últimos textos como Brewer (13), en 1953, quien escribe en su libro que "...la vulvo-vaginitis micótica es causa relativamente frecuente de leucorrea", aunque en verdad dedica muy pocas líneas a su descripción. Nosotros estamos seguros de que su incidencia aumentará día a día, en especial si los ginecólogos y laboratoristas clínicos adquieren mejores conocimientos para su diagnóstico.

La causa primordial de este aumento alarmante de las diferentes formas de moniliasis (oral, intestinal, vaginal, etc.), no es ctra que la aparición de los antibióticos en la terapéutica humana. En 1951 Woods y col. (14) describían 25 casos de infecciones moriliásicas localizadas en orofaringe, esófago, intestino y pulmones, a.tribuyendo tal hecho a los cambios en la biosintesis de las vitaminas por la ingestión de antibióticos, y antes, en 1950, Harris (15) reportaba la aparición de cultivos positivos para Candida Albicans procedentes de material recogido en vulva, vagina, piel perianal y boca de pacientes con complicaciones resultantes de terapia con aureomicina y cloramfenicol.

En la América Latina los mexicanos Alvarez y González (16) $\in \mathrm{n}$ 1952, presentan una estadística de dos series de casos no seleccionados, una anterior a 1950, y otra de enero a agosto de 1951; anotan un aumento en la incidencia de vulvo-vaginitis moniliásica entre los dos grupos del 2.4\% al 24.6\%. Estudiaron 172 casos no seleccionados de leucorrea y pudieron identificar el organismo en 34 casos; de éstos, 31 habían recibido antibióticos; tan sólo en uno había embarazo.

Si por otra parte recordamos a Bernstine y Rakoff (3) en su magnifico tratado sobre infecciones vaginales, hallamos que del 5 al $10 \%$ de todas las embarazadas presentan infección clínica micótica (aunque los cultivos pueden ser positivos en el 30\% de esos casos), no es difícil considerar la magnitud del problema actual. Campbell (4), al estudiar 836 pacientes privadas consecutivas en 1950, encontró una positividad de cultivos para Monilia Albicans en el $15.9 \%$ de los casos, lo que demuestra una altísima incidencia, aunque este dato de laboratorio aislado sin material clínico pierde para nosotros gran parte de su valor. 
En nuestro medio no hay referencias de mayor importancia. Gómez Vesga (17) sin embargo, presenta en 1954 un total de 1.704 estudios de laboratorio practicados en flujos fenitales, con una positividad para monilias en 60 casos, o sea el $3.5 \%$.

Deseo presentar mi estadística privada obtenida de julio de 1952 a julio de 1954 . Se trata de un total de 215 casos de mujeres que consultaron por flujo genital, o al menos éste era uno de los sintomas predominantes. En 51 casos se identificó la monilia como causa etiológica, de acuerdo con la clínica; en la misma serie, 50 casos fueron debidos a Tricomonas Vaginales, y tan sólo 6 a gonococo; uno por asociación, 2 por bacilos diftéricos, uno por neumococos, y el resto debido a causas no específicas.

En resumen:

$$
\begin{array}{cccc}
\text { Número de pacientes } & \text { Moniliasis } & \text { Tricomoniasis } & \text { Gonococcia } \\
215 & 51=23.7 \% & 50=23.2 \% & 6=2.8 \%
\end{array}
$$

De los 51 casos con moniliasis vaginal, 11 estaban embarazadas, 2 eran diabéticas y en 9 habia asociación con tricomonas.

En relación con antibióticos éste es el detalle, teniendo en cuenta su aplicación cercana previa a la aparición de la sintomatologia clínica:

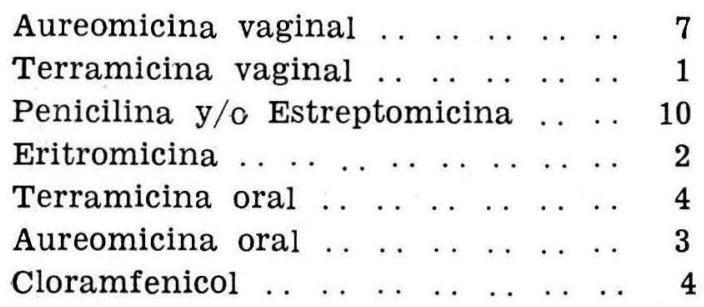

En total: 31 casos, o sea el $60 \%$.

Debo advertir que por tratarse de clientela privada de buenas condiciones económicas en general, y siendo en nuestro país libre la venta de antibióticos sin prescripción médica, podría afirmar que el $100 \%$ de esas mujeres habían recibido antibióticos en una o más ocasiones en el curso de los últimos cinco años. Por esta misma razón es probable que la incidencia de moniliasis vulvo-vaginal sea menor en clientela de tipo hospitalario. Por razones similares, sociales y económicas, podria interpretarse la baja incidencia de gonococcia genital femenina en la serie personal presentada. 
Como datos comparativos, no podría presentar series completas anteriores a 1952, pues en ese entonces no contaba como ahora con un laboratorio anexo y con un laboratorista especialmente entrenado para este tipo de investigación; pero la revisión aproximada no llega en ningún caso al $2 \%$.

\section{III. - APUNTES SOBRE TRATAMIENTO}

Por tratarse de una entidad considerada como rara hasta hace pocos años, pero cuya incidencia se encuentra en notable aumento por el uso intensivo de los antibióticos, a lo cual no escapan nuestras estadísticas, he considerado de importancia hacer a.lgunas anotaciones sobre su tratamiento.

Como lo advierten Bernstein y Rakoff (3), cuatro puntos vitales deben tenerse en cuenta:

1. Alivio del intenso prurito.

2. Ataque del organismo causal.

3. Producción en la vagina de un medio desfavorable a la multiplicación de tal organismo.

4. Prevención de la diseminación a otros lugares.

Debe tenerse en cuenta que, en micosis como la Moniliasis vaginal, no hay droga específica. Sin embargo hay un grupo de medicamentos reportados como altamente benéficos. El objeto de la presente comunicación es hacer un resumen de éstos, presentando algunas experiencias personales.

La mejor manera de aliviar rápidamente el prurito es iniciar pronto el tratamiento. La acción antipruriginosa de todos los mecicamentos que luego se mencionan parece hacerse evidente desde la primera o segunda aplicación. Si a pesar de esto el ardor y el prurito son muy molestos, la siguiente fórmula es recomendada:

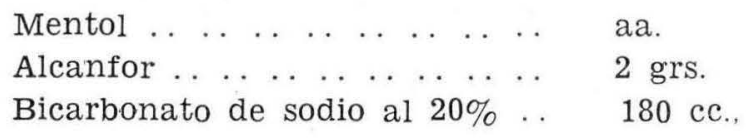

usando una cucharada en medio litro de agua tibia, para irrigaciones vaginales. Personalmente he obtenido buenos resultados con pomada de Nupercaína (Nupercainal Ciba), mejor quizás con ungüentos a base de Calamina (Caligesic), y superiores con ungüentos de hidrocortisona (Cortef "Upjohn"), para aplicación en 
las zonas vulvares y perivulvares; esto porque son los sitios en donde el prurito se hace notorio y a veces intolerable.

El ataque al organismo causal se inicia con una limpieza completa de la vagina; se aconsejan, luego de aplicar el espéculo vaginal, torundas con solución salina o bicarbonatada al 5\%; he usado en ocasiones la solución denominada "Cepacol" (Merrell), con buenos resultados.

En cuanto a los medicamentos de acción fungicida, he aquí una revisión resumida de los más usados:

1. Violeta de genciana.-- Es la droga que podríamos llamar clásica en el tratamiento de las micosis, y a mi modo de ver, sigue siendo si no la mejor, al menos no inferior a otras más modernas. Empleada en solución acuosa al $2 \%$ y cada tercer día, durante 2 o 3 semanas, barnizando toda la mucosa vaginal e incluyendo la vulva y regiones vecinas inmediatas, ha sido el sistema empleado por mí en un total de 74 casos tratados en la consulta privada de julio de 1952 a marzo de 1955. En la piel, y en casos rebeldes, aplico solución alcohólica de violeta de genciana al 5\%. cuidando de no tocar las mucosas, aun cuando de unos meses vara acá la he substituido por tintura de diclorhidrato de Diamthazol al 5\% en alcohol isopropílico al 70\%, por manchar menos y dar resultados similares. Los resultados finales puedo resumirlos así:

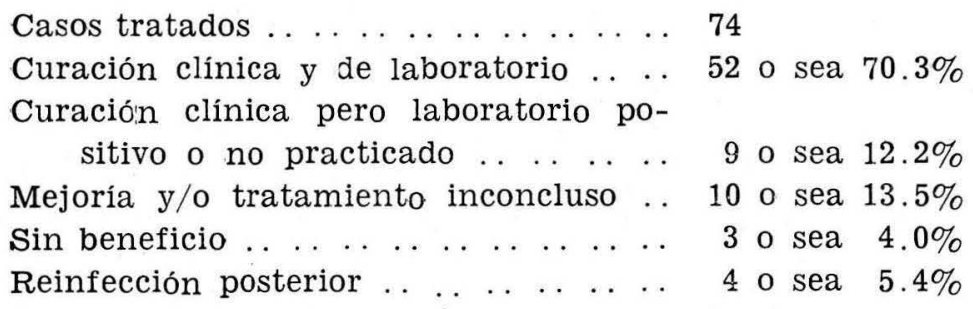

Ahora bien: si se considera como "curación" la clínica aunque no se practique el laboratorio - Hesseltine y Beckette (5), Hart y Brown (6) - el porcentaje de curación en los casos presentados alcanza el $82.5 \%$, cifra que es francamente similar a las que se verán luego. Es de hacer resaltar que por tratarse de una consulta privada me ha sido posible revisar un gran número de estos casos durante meses y aun años. De los tres fracasos, una diabética vieja que a pesar de no curar sí experimentó alivio, y dos embarazadas que no mejoraron sino al terminar el puer- 
perio; en una de ellas se usaron casi todos los medicamentos que se enunciarán posteriormente, sin resultados definitivos.

Como inconvenientes de la violeta de genciana en solución pueden recordarse: incomodidad de aplicación, pues debe hacerla exclusivamente el médico, y manchas en la ropa y material empleados, dificiles de quitar y realmente molestas para médico y paciente.

Las jaleas de violeta de genciana manchan un poco menos que las soluciones y son aplicables por la misma paciente. Waters y Wager (18) reportan en 191 casos de mujeres embarazadas sufriendo micosis vaginales y tratadas con el preparado conocido como "Gentian-Jel" (Westwood Pharmaceuticals), curación en el $78 \%$, mejoría en el $15 \%$ y fracasos en el $7 \%$, usando en el $87 \%$ de los casos "curados" tratamiento de dos semanas y 12 dosis en total. $\mathrm{Ni}$ esta jalea, ni otra de fabricación reciente, "Gentersal" (Ortho), se consiguen en nuestros mercados.

2. Iodo.- Su acción fungicida es reconocida desde hace muchos años. Se aconseja en solución de Lugol diluída del 1:4 al $1: 10$. Su aceptación hoy en día es escasa, y para lograr resultados convenientes habría que aplicarlos por largos períodos de tiempo. La he usado en muy pocos casos, sin éxito.

3. Acido caprílico.-- En 1953, Reich y col (19) reportan muy buenos resultados con el producto denominado "Naprilate" ( $R$. J. Satrassenburg Co.), aconsejando el siguiente esquema:

a) Lavado de la vagina con solución diluída de Caprilato de Sodio (una parte en 3 de agua);

b) Aplicación del polvo vaginal;

c) Crema intravaginal y también en toda la superficie adyacente externa;

d) Duchas caseras con una solución al $20 \%$ de caprilato de sodio, dos cucharadas en un litro de agua.

El tratamiento lo haria el médico en su consulta dos veces a la semana y la paciente en su casa (puntos c y d) 5 veces a la semana, en total por un tiempo de 5 semanas.

De 124 casos así tratados, los aa. reportan 93 con tratamiento completo y $86.2 \%$ de curaciones luego de 3 cultivos negativos. Es sin duda ninguna el mejor porcentaje de curaciones que encuentro en la revisión de la literatura a mi alcance; sin embargo, obsérvese la intensidad del tratamiento. El producto no ha sido importado a nuestro pais hasta el momento. 
4. Acido propiónico.-- Es tal vez la droga más popular para el tratamiento de moniliasis vulvo-vaginal en los Estados Unidos de Norteamérica, desde el trabajo de Alter y col. (20); Kalter (21) también usando la jalea "Propion-Gel" (Wyeth Inc.) en enfermas embarazadas, obtuvo mejoría en sus pacientes en el $75 \%$ de los casos y cultivos negativos en el $90 \%$ después de seis semanas consecutivas al parto; el autor aconseja aplicación nocturna de la jalea durante 2 a 3 semanas.

En los dos casos de pacientes embarazadas enunciados en mis clatos con fracaso al tratamiento por violeta de genciana, fue usado el Propion-Gel con mejoría apreciable del sintoma prurito, pero con recidiva al cesar la aplicación.

El producto tampoco se encuentra en nuestro comercio.

5. Diclorofeno.- Basándose en el buen poder fungicida de esta droga, la casa Frosst lanzó al mercado unos óvulos vaginales. “Trulfa-Caps". Inicié una pequeña experiencia en 5 casos no tratados previamente, siguiendo el esquema aconsejado: un óvulo a mañana y noche durante 9 días. Todas las pacientes acusaron irritación vaginal, y en dos fue tan intensa que se suspendió el tratamiento, continuándolo luego con violeta de genciana. En ctros dos no se obtuvo ningún resultado, y tan sólo en una se logró “curación". Francamente, no me parece aconsejable y no he podido encontrar referencias favorables.

6) Tri-Va.- Este preparado usado entusiásticamente por Gernard y Gallagher (22) en la tricomoniasis vaginal, fue empleado por los aa. en 15 casos de vulvo-vaginitis moniliásica con curas aparentes en $75 \%$ de las enfermas. Por tratarse de una serie pequeña, prometen continuar la investigación y dar a conocer sus resultados. Estos no han aparecido hasta el momento, año y medio después de la publicación inicial.

7. Diamthazol.- Es un derivado benzotiazolado que prepara Hoffmann La Roche, Inc., con la marca de fábrica "Asterol" en USA, y "Atelor" en Colombia. Henriksen y col. (7) reportan en septiembre de 1954 los siguientes resultados obtenidos con una crema vaginal experimental al 5\%, aplicándola 1-2 veces al día según irritación y durante seis semanas, sin importar que la enferma esté o no embarazada.

De 65 pacientes estudiadas, 83.1\% "curaron" (siguiendo las normas de Hesseltine y Breckette, etc., ya citados, quienes no consideran práctico establecer la curación por medio de cultivos 
regativos), $6.2 \%$ mejoraron y $10.7 \%$ no recibieron beneficio. Los aa. notaron recurrencia de vaginitis en 16 pacientes, pero tan solo en 4 se determinó la presencia de monilias. No observaron toxicidad ni en las pacientes ni en los niños de las embarazadas en tratamiento.

Por tratarse de una crema experimental no comercializada, pensé posible ensayar una solución muy diluída de la tintura (1 x 10), pero en las pocas ocasiones en que lo intenté, los tres casos fracasados a la violeta de genciana, la irritación fue tan intensa que no hizo posible su uso en la mucosa vaginal.

En cambio, como ya lo advertí, empleo en la actualidad la tintura en la región perivulvar en lugar de violeta de genciana en solución acuosa o alcohólica, con excelentes resultados al parecer.

Para terminar estos apuntes es bueno recordar que algunos autores (23), han observado que las jaleas contraceptivas parece que hacen menos frecuente la moniliasis en las mujeres que las usan; por eso, en ocasiones aconsejo una de tales jaleas para usar cierto tiempo después de terminar el tratamiento con violeta de genciana.

Por último, no olvidar examinar al marido, en especial en casos de reinfección posterior al tratamiento.

\section{SUMARIO}

Se presenta una discusión sobre criterios de diagnósticos y curación en moniliasis vaginal.

La clínica predomina ampliamente sobre el laboratorio.

En la práctica basta el diagnóstico de la especie monilia, sin importar mayor cosa el del género.

El criterio clínico predomina también en lo referente a curación, y una paciente puede ser declarada "clínicamente curada" a pesar de encontrarse el laboratorio positivo, si exámenes vaginales repetidos son normales y no hay sintomatología por un período no menor de un mes.

La moniliasis vulvo-vaginal presenta una incidencia alta en nuestro medio.

Se presenta una estadística privada de 215 casos con flujo genital, de los cuales 51 fueron positivos para moniliasis, o sea el $23.7 \%$; la tricomoniasis se encontró en $23.2 \%$ y la gonococcia en $2.8 \%$. 
La administración de antibióticos en época cercana previa a la aparición de los síntomas coincidió en el $60 \%$ de los casos estudiados de moniliasis vulvo-vaginal.

No hay droga específica contra la moniliasis vulvo-vaginal.

Los medicamentos mejores parecen ser: violeta de genciana, ácido caprílico, ácido propiónico y diamthazol.

Los resultados obtenidos con estas drogas parecen ser sensiblemente similares.

El autor presenta su experiencia personal en un grupo de pacientes privadas tratadas con soluciones de violeta de genciana, obteniendo curación clínica y de laboratorio en el $70.3 \%$, y “curación” clínica en un total de $82.5 \%$.

\section{REFERENCIAS}

1. DUbos R. J.- Bacterial and Mycotic Infections of Man. J. B. Lippincott Co., 1948, p. 601.

2. SWARTZ J. H.- Elements of Medical Mycology. Grune and Straton, 1949, p. 45.

3. BERNSTINE J. B., RAKOFF A. E.- Vaginal Infections, Infestations, and Discharges. The Blakiston Co., Inc., 1953.

4. CAMPBELL R. M., PARROTT M. H.- Vulvovaginal Moniliasis, Am. J. Obst. Gyn., 59: 1005, 1950.

5. HESSELTINE H. C., BECKETTE E. S.- Specific Treatment of Vaginal Mycosis. Am. J. Obst. Gyn. 58: 553, 1949.

6. HART D. M., BROWN C. H.- Brit. M. J., 2: 813, 1952.

7. HENRIKSEN E., MARTINS S. M., WILSON J. W., YEAMAN A.- Vaginitis due to Candida (Moniliasis) treated with a Benzothiazole derivative. Am. J. Obst. Gyn. 68: 830, 1954.

8. WILKINSON (cit. por Bernstine y Rakoff).

9. BLAND P. B., RAKOFF A. E., PINKUS I. J. -Experimental Vaginal and Cutaneous Moniliasis. Arch. Dermat. Syph., 36: 760, 1937.

10. HESSELTINE H. C.- Experimental and Clinical Therauy of Vulvo-Vaginal Mycosis. Amj. J. Obst. Gyn. 34: 439, 1937.

11. WHARTON L. R.- Gynecology with a Section on Female Urology. W. B. Saunders Co., 1944.

12. CURTIS A. H.- Gynecology. The University Society Inc., 1941.

13. BREWER J. I.- Textbook of Gynecology. The Williams and Wilkins Co., 1953.

14. 14. WOODS J. W., MANNING I. H., PATTERSON C. N.- Monilial Infections complicating the therapeutic use of antibiotics. J. A. M. A., 145: 207, 1951.

15. HARRIS H. J.- Aureomycin and Chloramphenicol in Brucellosis, with Special Reference to Side Effects. J. A. M. A., 142: 161, 1950. 
16. Alvarez B. A., GONZALEZ R. M.- Moniliasis Vaginal Consecutiva al uso terapéutico de antibióticos. Obst. Gin. Lat. Amer. 10: 295, 1952.

1\%. GOMEZ VESGA H.- Algunas consideraciones sobre los flujos genitales. Rev. Col. Obst. Gin. 5: 259, 1954.

18. WATERS E. G., WAGER H. P.- Vaginal Mycosis in Pregnancy; an Improved Gentian Violet Treatment. Am. J. Obst. Gyn., 60: 885, 1950.

10. REICH W. J., NECHTOW M. J., KURZON A. M., SUBOTNIK N., REICH J. B.- The Treatment of Monilial Vaginitis with Caprilic Acid. Am. J. Obst. Gyn., 65: 180, 1953.

20. Alter R. L., JONES C. P., CARTER B.- The Treatment of Mycotic Vulvovaginitis with Propionate Vaginal Jelly. Am. J. Obst. Gyn., 53: 241, 1947.

21. KANTOR H. I.- Evaluation of Treatment of Monilia in Pregnancy. Am. J. Obst. Gyn., 62: 170, 1951.

22. GERNAND H. C., GALLAGHER R.- Treatment of Vaginitis with "TriVa", Preliminary Report. Obst. \& Gyn., 2: 522, 1953.

22. STRAUSS H.- Gynecology and Obstetrics (C. H. Davis). W. F. Prior Co., 1953-1954. Vol. II. Cap. 17, p. 107. 